\title{
MATRIX WEIGHTED TRIEBEL-LIZORKIN BOUNDS: A SIMPLE STOPPING TIME PROOF
}

\author{
JOSHUA ISRALOWITZ
}

\begin{abstract}
In this paper we will give a simple stopping time proof in the $\mathbb{R}^{d}$ setting of the matrix weighted Triebel-Lizorkin bounds proved by F. Nazarov/S. Treil and A. Volberg, respectively. Furthermore, we provide explicit matrix $\mathrm{A}_{p}$ characteristic dependence and also discuss some interesting open questions.
\end{abstract}

\section{INTRODUCTION}

For any dyadic grid $\mathscr{D}$ in $\mathbb{R}$ and any interval in this grid, let

$$
h_{I}^{1}=|I|^{-\frac{1}{2}} \chi_{I}(x), \quad h_{I}^{0}(x)=|I|^{-\frac{1}{2}}\left(\chi_{I_{\ell}}(x)-\chi_{I_{r}}(x)\right) .
$$

Now given any dyadic grid $\mathscr{D}$ in $\mathbb{R}$, a cube $I=I_{1} \times \cdots \times I_{d}$, and any $\varepsilon \in\{0,1\}^{d}$, let $h_{I}^{\varepsilon}=\prod_{i=1}^{d} h_{I_{i}}^{\varepsilon}$. It is then easily seen that $\left\{h_{I}^{\varepsilon}\right\}_{I \in \mathscr{D}, \varepsilon \in \operatorname{Sig}_{d}}$ where $\operatorname{Sig}_{d}=\{0,1\}^{d} \backslash\{\overrightarrow{1}\}$ is an orthonormal basis for $L^{2}\left(\mathbb{R}^{d}\right)$.

The classical dyadic Littlewood-Paley estimates state that the Haar basis above is an unconditional basis for $L^{p}\left(\mathbb{R}^{d}\right)$ when $1<p<\infty$, and furthermore,

$$
\|f\|_{L^{p}} \approx\left(\int_{\mathbb{R}^{d}}\left(\sum_{I \in \mathscr{D}} \sum_{\varepsilon \in \operatorname{Sig}_{d}} \frac{\left|f_{I}^{\varepsilon}\right|^{2}}{|I|} \chi_{I}(x) d x\right)^{\frac{p}{2}} d x\right)^{\frac{1}{p}} .
$$

Now let $w$ be an $\mathrm{A}_{p}$ weight for $1<p<\infty$, meaning that

$$
\sup _{\substack{I \subseteq \mathbb{R}^{d} \\ I \text { is a cube }}}\left(\frac{1}{|I|} \int_{I} w(x) d x\right)\left(\frac{1}{|I|} \int_{I} w^{1-p^{\prime}}(x) d x\right)^{p-1}<\infty .
$$

The $L^{p}(w)$ version of (1.1) first proved in [4] states that

$$
\|f\|_{L^{p}(w)} \approx\left(\int_{\mathbb{R}^{d}}\left(\sum_{I \in \mathscr{D}} \sum_{\varepsilon \in \operatorname{Sig}_{d}} \frac{\left(m_{I} w\right)^{\frac{2}{p}}\left|f_{I}^{\varepsilon}\right|^{2}}{|I|} \chi_{I}(x) d x\right)^{\frac{p}{2}} d x\right)^{\frac{1}{p}}
$$

where $m_{I} w$ is the average of $w$ over $I$. Note that this says that $L^{p}(w)$ can be identified as a certain Triebel-Lizorkin space associated to the sequence $\left\{\left(m_{I} w\right)^{\frac{1}{p}}\right\}_{I \in \mathscr{D}}$ (see [4] for definitions).

2010 Mathematics Subject Classification. 42B20.

Key words and phrases. weighted norm inequalities, matrix weights. 
Now let $n \in \mathbb{N}$ and let $W: \mathbb{R}^{d} \rightarrow \mathcal{M}_{n}(\mathbb{C})$ be positive definite a. e. (where as usual $\mathcal{M}_{n}(\mathbb{C})$ is the algebra of $n \times n$ matrices with complex scalar entries). Define $L^{p}(W)$ to be the space of measurable functions $\vec{f}: \mathbb{R}^{d} \rightarrow \mathbb{C}^{n}$ with norm

$$
\|\vec{f}\|_{L^{p}(W)}^{p}=\int_{\mathbb{R}^{d}}\left|W^{\frac{1}{p}}(x) \vec{f}(x)\right|^{p} d x .
$$

Moreover, assume $W$ is a matrix $\mathrm{A}_{p}$ weight, meaning that

$$
\sup _{\substack{I \subset \mathbb{R}^{d} \\ I \text { is a cube }}} \frac{1}{|I|} \int_{I}\left(\frac{1}{|I|} \int_{I}\left\|W^{\frac{1}{p}}(x) W^{-\frac{1}{p}}(t)\right\|^{p^{\prime}} d t\right)^{\frac{p}{p^{\prime}}} d x<\infty
$$

where $p^{\prime}$ is the conjugate exponent of $p$. Note that matrix $\mathrm{A}_{p}$ weights arise naturally in various contexts and have drawn a lot of attention recently (see [1, 3, 7, 8, 10, [13 15] for example.)

A challenging question (definitively answered in [10,15] when $d=1$ ) is whether (1.2) can be extended to the matrix $\mathrm{A}_{p}$ setting, and if so what the "Littlewood-Paley expression" would look like. To answer this requires some more notation. It is well known (see [5] for example) that for a matrix weight $W$, a cube $I$, and any $1<p<$ $\infty$, there exists positive definite matrices $V_{I}$ and $V_{I}^{\prime}$ such that $|I|^{-\frac{1}{p}}\left\|\chi_{I} W^{\frac{1}{p}} \vec{e}\right\|_{L^{p}} \approx$ $\left|V_{I} \vec{e}\right|$ and $|I|^{-\frac{1}{p^{\prime}}}\left\|\chi_{I} W^{-\frac{1}{p}} \vec{e}\right\|_{L^{p^{\prime}}} \approx\left|V_{I}^{\prime} \vec{e}\right|$ for any $\vec{e} \in \mathbb{C}^{n}$, where $\|\cdot\|_{L^{p}}$ is the canonical $L^{p}\left(\mathbb{R}^{d} ; \mathbb{C}^{n}\right)$ norm and the notation $A \approx B$ as usual means that two quantities $A$ and $B$ are bounded above and below by a constant multiple of each other (which unless otherwise stated will not depend on the weight $W$ ). Note that it is easy to see that $\left\|V_{I} V_{I}^{\prime}\right\| \geq 1$ for any cube $I$. We will say that $W$ is a matrix $\mathrm{A}_{p}$ weight if the product $V_{I} V_{I}^{\prime}$ has uniformly bounded matrix norm with respect to all cubes $I \subset \mathbb{R}^{d}$ (note that this condition is easily seen to be equivalent to (1.3), see p. 275 in [13] for example.) Also note that when $p=2$ we have $V_{I}=\left(m_{I} W\right)^{\frac{1}{2}}$ and $V_{I}^{\prime}=\left(m_{I}\left(W^{-1}\right)\right)^{\frac{1}{2}}$ where $m_{I} W$ is the average of $W$ on $I$, so that the matrix $\mathrm{A}_{2}$ condition takes on a particularly simple form that is similar to the scalar $\mathrm{A}_{2}$ condition.

Let us emphasize to the reader that while the matricies $V_{I}$ and $V_{I}^{\prime}$ are not averages of $W$, it is nonetheless very useful to think of them as appropriate averages. With this in mind, it was proved in 10,15 , that the correct matrix weighted version of (1.2) is as follows: if $1<p<\infty$ and $W$ is a matrix $\mathrm{A}_{p}$ weight on $\mathbb{R}$, then for any $\vec{f} \in L^{p}(W)$ we have

$$
\|\vec{f}\|_{L^{p}(W)} \approx\left(\int_{\mathbb{R}}\left(\sum_{I \in \mathscr{D}} \frac{\left|V_{I} \vec{f}_{I}\right|^{2}}{|I|} \chi_{I}(x)\right)^{\frac{p}{2}} d x\right)^{\frac{1}{p}}
$$

where $\vec{f}_{I}$ is the vector of Haar coefficients of the components of $\vec{f}$ with respect to I. Note that (1.4) has a number of applications to operators related to singular integral operators (see [1,7, 8, 10, 15, for example) and in some sense has the effect of allowing one to "remove" $W$ and replace it with the $V_{I}^{\prime} s$. Furthermore, while is it probably likely that the arguments in [15] can be extended to $\mathbb{R}^{d}$, [10] primarily 
involves Bellman function arguments, which are nontrivial (though not necessarily difficult) to extend to $\mathbb{R}^{d}$.

The purpose of this paper is to provide a simple stopping time proof of (1.4) for $\mathbb{R}^{d}$ that, unlike [10,15], closely resembles known scalar techniques, and in particular is a modification of the stopping time techniques from 9] (note that this modification was done in the $p=2$ setting in [11] for operator valued weights). Moreover, we also track the $A_{p}$ characteristic in (1.4). In particular, we will precisely prove the following, where here $\lceil p\rceil$ is the smallest integer greater or equal to $p$.

Theorem 1.1. If $1<p<\infty$ and $W$ is a matrix $A_{p}$ weight then the following two inequalities hold for any $\vec{f} \in L^{p}(W)$ :

$$
\|\vec{f}\|_{L^{p}(W)} \lesssim\|W\|_{A_{p}}^{\frac{1}{p}+\frac{\lceil p]}{p}}\left(\int_{\mathbb{R}^{d}}\left(\sum_{I \in \mathscr{D}} \sum_{\varepsilon \in \operatorname{Sig}_{d}} \frac{\left|V_{I} \vec{f}_{I}^{\varepsilon}\right|^{2}}{|I|} \chi_{I}(x)\right)^{\frac{p}{2}} d x\right)^{\frac{1}{p}}
$$

and

$$
\left(\int_{\mathbb{R}^{d}}\left(\sum_{I \in \mathscr{D}} \sum_{\varepsilon \in S i g_{d}} \frac{\left|V_{I} \vec{f}_{I}^{\varepsilon}\right|^{2}}{|I|} \chi_{I}(x)\right)^{\frac{p}{2}} d x\right)^{\frac{1}{p}} \lesssim\|W\|_{A_{p}}^{\frac{2}{p}+\frac{\left\lceil p^{\prime}\right]}{p}}\|\vec{f}\|_{L^{p}(W)}
$$

Note that in some sense "half" of (1.4) was proved in [10,15, for $\mathrm{A}_{p, \infty}$ weights when $d=1$ (see either of these for definitions, and see Theorem 3.1 for the precise result). Furthermore, as we will explain in the last section, our proof, with the help of a Lemma 3.1 in [15] also proves this. Also, we will discuss what is known in the literature for the $\mathrm{A}_{2}$ dependence in (1.4) in the case $p=2$.

Finally, it is hoped that the "matrixizing" of the ideas in [9] for $p \neq 2$ will have applications to other challenging yet nonetheless interesting problems involving $L^{p}(W)$ where cancellation plays a key role. Furthermore, it would be very interesting to explore whether a Besov space version of Theorem 1.1] holds (see [13] for results similar to this but outside of the Haar realm.) In particular, note that Theorem 1.1 was instrumental in proving (in [8]) the equivalency between a dyadic and a continuous characterization of the boundedness of the commutators $\left[B, R_{j}\right]$ on $L^{p}(W)$ in terms of $B$ (for $B$ a locally integrable $\mathcal{M}_{n}(\mathbb{C})$ valued function and $R_{j}$ being any of the Riesz transforms). Therefore, it is obviously natural to expect that a Besov space version of Theorem 1.1 would help in proving Schatten $p$ class characterizations of $\left[B, R_{j}\right]$ on $L^{2}(W)$ in terms of $B$.

\section{MAin Lemma}

In this section we will prove a crucial lemma (Lemma 2.4). In fact, the proof of Theorem 1.1 will follow almost immediately from Lemma 2.4 and some elementary approximation and duality arguments. We will first introduce some stopping time definitions from [9] and prove some preliminary lemmas. For any dyadic grid $\mathscr{D}$ in $\mathbb{R}^{d}$ and $I \in \mathscr{D}$, let $\mathscr{J}(I)$ be the collection of dyadic cubes in $\mathscr{D}(I)$ that are maximal with respect to a certain property. Let $\mathscr{F}(I)$ be the collection of all dyadic cubes in $\mathscr{D}(I)$ that are not contained in any $J \in \mathscr{J}(I)$. We say the property is admissible 
if $I \in \mathscr{F}(I)$ for every $I \in \mathscr{D}$. Given an admissible property, let $\mathscr{J}^{0}(I):=I$ and for $j \in \mathbb{N}$ inductively let $\mathscr{J}^{j}(I)$ be the collection of cubes belonging to $\mathscr{J}(J)$ for some $J \in \mathscr{J}^{j-1}(I)$. Similarly define $\mathscr{F}^{j}(I)$ be the collection of cubes belonging to $\mathscr{F}(J)$ for some $J \in \mathscr{J}^{j-1}(I)$. Note that the admissible property clearly gives us that $\mathscr{D}(I)=\cup_{j=1}^{\infty} \mathscr{F}^{j}(I)$. Also clearly the $\mathscr{F}^{j}(I)$ 's are disjoint (with respect to $j$ ) collections of cubes. We will refer to the stopping time above by simply $\mathscr{J}$.

We will say the stopping time above is decaying if there exists $0<c<1$ such that

$$
\sup _{I \in \mathscr{D}} \frac{1}{|I|} \sum_{J \in \mathscr{J}(I)}|J| \leq c .
$$

Clearly by iteration this gives us that

$$
\sup _{I \in \mathscr{D}} \frac{1}{|I|} \sum_{J \in \mathscr{J}^{j}(I)}|J| \leq c^{j}
$$

Now given a decaying stopping time, fix some large cube $I_{0} \in \mathscr{D}$ and for notational simplicity let $\mathscr{F}^{j}=\mathscr{F}^{j}\left(I_{0}\right)$ and define $\mathscr{J}^{j}$ similarly. Also for some $\vec{f} \in L^{p}$ let

$$
\Delta_{j} \vec{f}=\sum_{I \in \mathscr{F}^{j}} \sum_{\varepsilon \in \operatorname{Sig}_{d}} \vec{f}_{I}^{\varepsilon} h_{I}^{\varepsilon}
$$

The proof of the following is exactly the same as the proof of Lemma 7 in [9] and therefore will be omitted.

Lemma 2.1. Let $\mathscr{J}$ be a decaying stopping time. For any $1<p<\infty$ we have that

$$
\sum_{j=0}^{\infty}\left\|\Delta_{j} \vec{f}\right\|_{L^{p}}^{p} \lesssim\|\vec{f}\|_{L^{p}}^{p}
$$

Note of course that the implicit constant above depends on the rate of decay of $\mathscr{J}$, but not on the cube $I_{0}$. As we will see later, the dependence on the decay rate is not important.

The proof of the next lemma is exactly the same as the proof of Lemma 8 in 9]. We will however provide a complete proof as we will need to carefully track the constants involved.

Lemma 2.2. Let $\mathscr{J}$ be a decaying stopping time and let $1<p<\infty$. Let $T$ be a linear operator on some class of measurable $\mathbb{C}^{n}$ valued functions and suppose that $T \vec{f}=\sum_{j=1}^{\infty} T_{j} \vec{f}$ pointwise a.e. for all $\vec{f}$ in this class, where $T_{j}=T \Delta_{j}$ and each $T_{j} \vec{f}$ is measurable. Assume that there exists $0<c<1$ and $C>0$ where

$$
\int_{\mathbb{R}^{d}}\left|T_{k} \vec{f}\right|^{\frac{p}{2}}\left|T_{j} \vec{f}\right|^{\frac{p}{2}} d x \leq C c^{|k-j|}\left\|\Delta_{j} \vec{f}\right\|_{L^{p}}^{\frac{p}{2}}\left\|\Delta_{k} \vec{f}\right\|_{L^{p}}^{\frac{p}{2}} .
$$

Then we have the bound

$$
\|T \vec{f}\|_{L^{p}}^{p} \lesssim \frac{C}{(1-c)^{\lceil p\rceil}}\|\vec{f}\|_{L^{p}}^{p}
$$

for any $\vec{f}$ in this class (where again the implicit constant above depends on the rate of decay of $\mathscr{J}$ but not on $I_{0}$.) 
Proof. Let $n=\lceil p\rceil$. Then by Jensen's inequality, Hölder's inequality, and the assumption above, we have

$$
\begin{aligned}
\int_{\mathbb{R}^{d}}|T \vec{f}|^{p} d x & \\
& \leq \int_{\mathbb{R}^{d}}\left(\sum_{j=1}^{\infty}\left|T_{j} \vec{f}\right|^{\frac{p}{n}}\right)^{n} d x \\
& =\sum_{\left(j_{1}, \ldots, j_{n}\right) \in \mathbb{N}^{n}} \int_{\mathbb{R}^{d}}\left|T_{j_{1}} \vec{f}\right|^{\frac{p}{n}} \cdots\left|T_{j_{n}} \vec{f}\right|^{\frac{p}{n}} d x \\
& \lesssim \sum_{j_{1} \leq j_{2} \leq \cdots \leq j_{n}} \int_{\mathbb{R}^{d}}\left|T_{j_{1}} \vec{f}\right|^{\frac{p}{n}} \cdots\left|T_{j_{n}} \vec{f}\right|^{\frac{p}{n}} d x \\
& \leq \sum_{j_{1} \leq j_{2} \leq \cdots \leq j_{n}}\left(\int_{\mathbb{R}^{d}}\left|T_{j_{1}} \vec{f}\right|^{\frac{p}{2}}\left|T_{j_{2}} \vec{f}\right|^{\frac{p}{2}} d x\right)^{\frac{1}{n}}\left(\int_{\mathbb{R}^{d}}\left|T_{j_{2}} \vec{f}\right|^{\frac{p}{2}}\left|T_{j_{3}} \vec{f}\right|^{\frac{p}{2}} d x\right)^{\frac{1}{n}} \cdots\left(\int_{\mathbb{R}^{d}}\left|T_{j_{n}} \vec{f}\right|^{\frac{p}{2}}\left|T_{j_{1}} \vec{f}\right|^{\frac{p}{2}} d x\right)^{\frac{1}{n}} \\
& \leq C \sum_{j_{1} \leq j_{2} \leq \cdots \leq j_{n}} c^{\frac{j_{2}-j_{1}}{n}} c^{\frac{j_{3}-j_{2}}{n}} \cdots c^{\frac{j_{n}-j_{1}}{n}}\left\|\Delta_{j_{1}} \vec{f}\right\|_{L^{p}}^{\frac{p}{n}} \cdots\left\|\Delta_{j_{n}} \vec{f}\right\|_{L^{p}}^{\frac{p}{n}}
\end{aligned}
$$

Using Hölder's inequality one more time and the previous Lemma, we get that

$$
\begin{aligned}
& \int_{\mathbb{R}^{d}}|T \vec{f}|^{p} d x \\
& \leq C\left(\sum_{j_{1} \leq j_{2} \leq \cdots \leq j_{n}} c^{\frac{j_{2}-j_{1}}{n}} c^{\frac{j_{3}-j_{2}}{n}} \cdots c^{\frac{j_{n}-j_{1}}{n}}\left\|\Delta_{j_{1}} \vec{f}\right\|_{L^{p}}^{p}\right)^{\frac{1}{n}} \\
& \quad \times \cdots \times\left(\sum_{j_{1} \leq j_{2} \leq \cdots \leq j_{n}} c^{\frac{j_{2}-j_{1}}{n}} c^{\frac{j_{3}-j_{2}}{n}} \cdots c^{\frac{j_{n}-j_{1}}{n}}\left\|\Delta_{j_{n}} \vec{f}\right\|_{L^{p}}^{p}\right)^{\frac{1}{n}} \\
& \quad \frac{C}{(1-c)^{\lceil p\rceil}} \sum_{j=1}^{\infty}\left\|\Delta_{j} \vec{f}\right\|_{L^{p}}^{p} \\
& \quad \lesssim \frac{C}{(1-c)^{\lceil p\rceil}}\|\vec{f}\|_{L^{p}}^{p} .
\end{aligned}
$$

Now we will describe our precise stopping time. For any cube $I \in \mathscr{D}$, let $\mathscr{J}(I)$ be the collection of maximal $J \in \mathscr{D}(I)$ such that

$$
\left\|V_{J} V_{I}^{-1}\right\|^{p}>\lambda_{1} \text { or }\left\|V_{J}^{-1} V_{I}\right\|^{p^{\prime}}>\lambda_{2}
$$

for some $\lambda_{1}, \lambda_{2}>1$ to be specified later. Also, clearly $J \in \mathscr{F}(J)$ for any $J \in \mathscr{D}(I)$ so that $\mathscr{J}$ is an admissible stopping time.

We will now show that $\mathscr{J}$ is decaying.

Lemma 2.3. Let $1<p<\infty$ and let $W$ be a matrix $A_{p}$ weight. For $\lambda_{1}, \lambda_{2}>1$ large enough, we have that $\left|\bigcup \mathscr{J}^{j}(I)\right| \leq 2^{-j}|I|$ for every $I \in \mathscr{D}$. 
Proof. By iteration, it is enough to prove the lemma for $j=1$. For $I \in \mathscr{D}$, let $\mathscr{G}(I)$ denote the collection of maximal $J \in \mathscr{D}(I)$ such that the first inequality (but not necessarily the second inequality) in (2.1) holds. Then by maximality and elementary linear algebra, we have that

$$
\left|\bigcup_{J \in \mathscr{G}(I)} J\right|=\sum_{J \in \mathscr{G}(I)}|J| \lesssim \frac{1}{\lambda_{1}} \sum_{J \in \mathscr{G}(I)} \int_{J}\left\|W^{\frac{1}{p}}(y) V_{I}^{-1}\right\|^{p} d y \leq \frac{C_{1}|I|}{\lambda_{1}}
$$

for some $C_{1}>0$ only depending on $n$ and $d$.

On the other hand, let For $I \in \mathscr{D}$, let $\widetilde{\mathscr{G}}(I)$ denote the collection of maximal $J \in \mathscr{D}(I)$ such that the second inequality (but not necessarily the first inequality) in (2.1) holds. Then by the matrix $\mathrm{A}_{p}$ condition we have

$$
\left|\bigcup_{J \in \widetilde{\mathscr{G}}(I)} J\right| \leq \frac{C_{2}}{\lambda_{2}} \sum_{J \in \widetilde{\mathscr{G}}(I)} \int_{J}\left\|W^{-\frac{1}{p}}(y) V_{I}\right\|^{p^{\prime}} d y \leq \frac{C_{2}^{\prime}\|W\|_{A_{p}}^{\frac{p^{\prime}}{p}}}{\lambda_{2}}|I|
$$

for some $C_{2}^{\prime}$ only depending on $n$ and $d$. The proof is now completed by setting $\lambda_{1}=4 C_{1}$ and $\lambda_{2}=4 C_{2}^{\prime}\|W\|_{A_{p}}^{\frac{p^{\prime}}{p}}$.

While we will not have a need to discuss matrix $\mathrm{A}_{p, \infty}$ weights in detail in this paper, note that in fact Lemma 3.1 in $[15$ immediately gives us that Lemma 2.3 holds for matrix $\mathrm{A}_{p, \infty}$ weights (with possibly larger $\lambda_{2}$ of course.)

Let $\mathcal{F}$ be the vector space of all $\vec{f} \in \mathscr{F}$. Now define the constant Haar multiplier $M_{W, p}$ by

$$
M_{W, p} \vec{f}:=\sum_{I \in \mathscr{D}} \sum_{\varepsilon \in \operatorname{Sig}_{d}} V_{I} \vec{f}_{I}^{\varepsilon} h_{I}^{\varepsilon} .
$$

which obviously is defined on $\mathcal{F}$ and define $M_{W, p}^{-1}$ on $\mathcal{F}$ in the obvious way. We can now state and prove the main result of this section.

Lemma 2.4. Let $1<p<\infty$. If $W$ is a matrix $A_{p}$ weight, then $W^{\frac{1}{p}} M_{W, p}^{-1}$ satisfies the bounds

$$
\left\|W^{\frac{1}{p}} M_{W, p}^{-1} \vec{f}\right\|_{L^{p}}^{p} \lesssim\|W\|_{A_{p}}^{1+\lceil p\rceil}\|\vec{f}\|_{L^{p}}^{p}
$$

for all $\vec{f}$ with finite Haar expansion

Proof. Fix some $I_{0} \in \mathscr{D}$. Obviously it is enough to prove that the operator $T$ defined on $\mathscr{F}$ by

$$
T \vec{f}:=\sum_{I \in \mathscr{D}\left(I_{0}\right)} \sum_{\varepsilon \in \operatorname{Sig}_{d}} W^{\frac{1}{p}} V_{I}^{-1} \vec{f}_{I}^{\varepsilon} h_{I}^{\varepsilon}
$$

satisfies the above bound independent of $I_{0} \in \mathscr{D}$. Note that we also clearly have $T \vec{f}=\sum_{j=1}^{\infty} T_{j} \vec{f}$ for $\vec{f} \in \mathcal{F}$. Now fix some $\vec{f} \in \mathcal{F}$.

For each $I \in \mathscr{D}$, let

$$
M_{I} \vec{f}:=\sum_{J \in \mathscr{F}(I)} \sum_{\varepsilon \in \operatorname{Sig}_{d}} V_{J}^{-1} \vec{f}_{J}^{\varepsilon} h_{J}^{\varepsilon}
$$


so that

$$
T_{j} \vec{f}=\sum_{I \in \mathscr{J}^{j-1}} W^{\frac{1}{p}} M_{I} \vec{f}
$$

Since $V_{I} M_{I}$ is a constant Haar multiplier and since $\left\|V_{I} V_{J}^{-1}\right\|^{p} \lesssim\|W\|_{\mathrm{A}_{p}}$ if $J \in$ $\mathscr{F}(I)$, we immediately have that

$$
\left\|V_{I} M_{I} \vec{f}\right\|_{L^{p}}^{p} \lesssim\|W\|_{A_{p}}\|\vec{f}\|_{L^{p}}^{p} .
$$

Now we will show that each $T_{j}$ is bounded. To that end, we have that

$$
\begin{aligned}
\int_{\mathbb{R}^{d}}\left|T_{j} f\right|^{p} d x & =\int_{\bigcup_{\mathscr{J}^{j-1}} \backslash \bigcup_{\mathscr{J}^{j}}}\left|T_{j} f\right|^{p} d x+\int_{\bigcup_{\mathscr{J}^{j}}}\left|T_{j} f\right|^{p} d x \\
& :=(A)+(B) .
\end{aligned}
$$

Since $\left\|W^{\frac{1}{p}}(x) V_{J}^{-1}\right\|^{p} \lesssim 1$ on $J \backslash \bigcup \mathscr{J}(J)$, we can estimate $(A)$ first as follows:

$$
\begin{aligned}
(A) & =\sum_{J \in \mathscr{J}^{j-1}} \int_{J \backslash \cup \mathscr{J}(J)}\left|T_{j} \vec{f}\right|^{p} d x \\
& =\sum_{J \in \mathscr{J}^{j-1}} \int_{J \backslash \cup \mathscr{J}(J)}\left|W^{\frac{1}{p}}(x) M_{J} \vec{f}(x)\right|^{p} d x \\
& \leq \sum_{J \in \mathscr{J}^{j-1}} \int_{J \backslash \cup \mathscr{J}(J)}\left\|W^{\frac{1}{p}}(x) V_{J}^{-1}\right\|^{p}\left|V_{J} M_{J} \vec{f}(x)\right|^{p} d x \\
& \lesssim \sum_{J \in \mathscr{J}^{j-1}} \int_{J}\left|V_{J} M_{J} \vec{f}\right|^{p} d x \\
& \lesssim\|W\|_{A_{p}}\|\vec{f}\|_{L^{p}}^{p} .
\end{aligned}
$$

As for $(B)$, note that $M_{J} \vec{f}$ is constant on $I \in \mathscr{J}(J)$, and so we will refer to this constant by $M_{J} \vec{f}(I)$. We then estimate $(B)$ as follows:

$$
\begin{aligned}
(B) & =\int_{\cup \mathscr{J}^{j}}\left|T_{j} \vec{f}\right|^{p} d x \\
& \leq \sum_{J \in \mathscr{J}^{j-1}} \sum_{I \in \mathscr{J}(J)} \int_{I}\left|W^{\frac{1}{p}}(x) M_{J} \vec{f}\right|^{p} d x \\
& \leq \sum_{J \in \mathscr{J}^{j-1}} \sum_{I \in \mathscr{J}(J)}|I|\left|V_{J} M_{J} \vec{f}(I)\right|\left(\frac{1}{|I|} \int_{I}\left\|W^{\frac{1}{p}}(x) V_{J}^{-1}\right\|^{p} d x\right) \\
& \lesssim \sum_{J \in \mathscr{J}^{j}-1} \sum_{I \in \mathscr{J}(J)}|I|\left|V_{J} M_{J} \vec{f}(I)\right| \\
& =\sum_{J \in \mathscr{J}^{j-1}} \sum_{I \in \mathscr{J}(J)} \int_{I}\left|V_{J} M_{J} \vec{f}(x)\right|^{p} d x \\
& \lesssim\|W\|_{A_{p}}\|\vec{f}\|_{L^{p}}^{p} .
\end{aligned}
$$


However, clearly $T_{j} \vec{f}=T_{j} \Delta_{j} \vec{f}$ so that

$$
\int_{\mathbb{R}^{d}}\left|T_{j} f\right|^{p} d x \lesssim\|W\|_{\mathrm{A}_{p}}\left\|\Delta_{j} \vec{f}\right\|_{L^{p}}^{p}
$$

To finish the proof, we claim that there exists $0<c<1$ such that

$$
\int_{\bigcup \mathscr{J}^{k-1}}\left|T_{j} \vec{f}\right|^{p} d x \lesssim c^{k-j}\left\|\vec{f}_{j}\right\|_{L^{p}}^{p}
$$

whenever $k>j$. If we define $M_{j} \vec{f}$ as

$$
M_{j} \vec{f}:=\sum_{I \in \mathscr{J}^{j-1}} M_{I} \vec{f}
$$

then $M_{j} \vec{f}$ is constant on $J \in \mathscr{J}^{j}$. Thus, we have that

$$
\begin{aligned}
\int_{\bigcup \mathscr{J}^{k-1}}\left|T_{j} \vec{f}\right|^{p} d x & =\sum_{J \in \mathscr{J}^{j}} \sum_{I \in \mathscr{J}^{k-j-1}(J)} \int_{I}\left|W^{\frac{1}{p}}(x) M_{j} \vec{f}(J)\right|^{p} d x \\
& \leq \sum_{J \in \mathscr{J}^{j}} \sum_{I \in \mathscr{J}^{k-j-1}(J)}|J|\left|V_{J} M_{j} \vec{f}(J)\right|^{p}\left(\frac{1}{|J|} \int_{I}\left\|W^{\frac{1}{p}}(x) V_{J}^{-1}\right\|^{p} d x\right) .
\end{aligned}
$$

However,

$$
\begin{aligned}
|J|\left|V_{J} M_{j} \vec{f}(J)\right|^{p} & \lesssim \int_{J}\left|W^{\frac{1}{p}}(x) M_{j} \vec{f}(J)\right|^{p} d x \\
& =\int_{J}\left|T_{j} \vec{f}(x)\right|^{p} d x .
\end{aligned}
$$

On the other hand, it is not hard to show that $\left|W^{\frac{1}{p}}(x) \vec{e}\right|^{p}$ is a scalar $\mathrm{A}_{p}$ weight for any $\vec{e} \in \mathbb{C}^{n}$ (see [5]), which by the classical reverse Hölder inequality means that we can pick some $q>p$ and use Hölder's inequality in conjunction with Lemma 2.3 to get

$$
\begin{aligned}
& \frac{1}{|J|} \sum_{I \in \mathscr{J}^{k-j-1}(J)} \int_{I}\left\|W^{\frac{1}{p}}(x) V_{J}^{-1}\right\|^{p} d x \\
&=\frac{1}{|J|} \int_{\bigcup \mathscr{J}^{k-j-1}(J)}\left\|W^{\frac{1}{p}}(x) V_{J}^{-1}\right\|^{p} d x \\
& \leq \frac{1}{|J|}\left(\int_{\bigcup \mathscr{J}^{k-j-1}(J)}\left\|W^{\frac{1}{p}}(x) V_{J}^{-1}\right\|^{q} d x\right)^{\frac{p}{q}}\left(2^{-(k-j-1)}|J|\right)^{1-\frac{p}{q}} \\
& \lesssim 2^{-(k-j-1)\left(1-\frac{p}{q}\right)}\left(\frac{1}{|J|} \int_{J}\left\|W^{\frac{1}{p}}(x) V_{J}^{-1}\right\|^{q} d x\right)^{\frac{p}{q}} \\
& \lesssim 2^{-(k-j-1)\left(1-\frac{p}{q}\right)} .
\end{aligned}
$$

Combining (2.3) with (2.4), we get that

$$
\begin{aligned}
\int_{\bigcup \mathscr{J}^{k-1}}\left|T_{j} \vec{f}\right|^{p} d x & \lesssim 2^{-(k-j-1)\left(1-\frac{p}{q}\right)} \int_{\bigcup_{\mathscr{J}^{j}}}\left|T_{j} \vec{f}\right|^{p} d x \\
& \lesssim\|W\|_{A_{p}} 2^{-(k-j-1)\left(1-\frac{p}{q}\right)}\left\|\Delta_{j} \vec{f}\right\|_{L^{p}}^{p} .
\end{aligned}
$$


Note however, that the scalar characteristic of each $\|W\||\vec{e}|$ is less than or equal to $\|W\|_{A_{p}}|\vec{e}|$ which means that classically we can choose $q$ such that

$$
q=p+\frac{1}{C\|W\|_{\mathrm{A}_{p}}}
$$

for some $C>1$ independent of $W$, so that

$$
2^{-\left(1-\frac{p}{q}\right)}=2^{-\frac{1}{q C\|W\|_{\mathrm{A}_{p}}}}=2^{-\frac{1}{p C\|W\|_{\mathrm{A}_{p}+1}}}
$$

which means that

$$
\int_{\bigcup \mathscr{J}^{k-1}}\left|T_{j} \vec{f}\right|^{p} d x \lesssim\|W\|_{\mathrm{A}_{p}} 2^{-\frac{k-j-1}{p C\|W\|_{\mathrm{A}_{p}+1}}}\left\|\Delta_{j} \vec{f}\right\|_{L^{p}}^{p}
$$

since again $T_{j} \vec{f}=T_{j} \Delta_{j} \vec{f}$.

Finally, this gives us that

$$
\begin{aligned}
\int_{\mathbb{R}^{d}}\left|T_{k} \vec{f}\right|^{\frac{p}{2}}\left|T_{j} \vec{f}\right|^{\frac{p}{2}} d x & \leq\left(\int_{\bigcup \mathscr{J}^{k-1}}\left|T_{j} \vec{f}\right|^{p} d x\right)^{\frac{1}{2}}\left\|T_{k} \vec{f}\right\|_{L^{p}}^{\frac{p}{2}} \\
& \lesssim\left(\|W\|_{\mathrm{A}_{p}} 2^{-\frac{k-j-1}{p C\|W\|_{\mathrm{A}_{p}+1}}}\left\|\Delta_{k} \vec{f}\right\|_{L^{p}}^{p}\right)^{\frac{1}{2}}\left(\|W\|_{\mathrm{A}_{p}}\left\|\Delta_{j} \vec{f}\right\|_{L^{p}}^{p}\right)^{\frac{1}{2}}
\end{aligned}
$$

and combining this with the previous two lemmas gives us that

$$
\begin{aligned}
\left\|W^{\frac{1}{p}} M_{W, p}^{-1} \vec{f}\right\|_{L^{p}}^{p} & \lesssim\|W\|_{\mathrm{A}_{p}}\left(\frac{1}{1-2^{-\frac{1}{2 p C\|W\|_{\mathrm{A}_{p}}}}}\right)^{\lceil p\rceil}\|\vec{f}\|_{L^{p}}^{p} \\
& \lesssim\|\vec{f}\|_{L^{p}}^{p}\|W\|_{\mathrm{A}_{p}}^{1+\lceil p\rceil}
\end{aligned}
$$

Note that the proof of Lemma 2.4 only requires Lemma 2.3 and the fact that $\left|W^{\frac{1}{p}}(x) \vec{e}\right|^{p}$ satisfies a reverse Hölder inequality for each $\vec{e}$. In particular, our proof (as do proofs in [10,15]) holds for matrix $\mathrm{A}_{p, \infty}$ weights.

\section{Proof of Theorem 1.1 and Comments}

We will first prove Theorem 1.1 for $C_{c}^{\infty}\left(\mathbb{R}^{d}\right)$ functions, which largely involves Lemma 2.4 in conjunction with some easy duality arguments. Theorem 1.1 in general will then follow from the fact that $C_{c}^{\infty}\left(\mathbb{R}^{d}\right)$ is dense in $L^{p}(W)$ (see Proposition 3.7 in [2, which interestingly holds for not necessarily matrix $\mathrm{A}_{p}$ weights) and some elementary approximation arguments. For the rest of this section, pick strictly increasing finite subsets $\left\{F_{k}\right\}_{k=1}^{\infty}$ of $\mathscr{D}$ whose union is all of $\mathscr{D}$.

Proof of Theorem 1.1. We first prove the first inequality in Theorem 1.1 for $C_{c}^{\infty}\left(\mathbb{R}^{d}\right)$ functions. Let $\vec{f} \in C_{c}^{\infty}\left(\mathbb{R}^{d}\right)$ and let $\vec{f}_{k}$ be defined by

$$
\overrightarrow{f_{k}}=\sum_{I \in F_{k}} \sum_{\varepsilon \in \operatorname{Sig}_{d}} \vec{f}_{I}^{\varepsilon} h_{I}^{\varepsilon}
$$


so that obviously $M_{W, p} \vec{f}_{k}$ is well defined. Also note that obviously we have $\mathscr{F} \subseteq$ $L^{p}(W)$. Then from Lemma 2.4 we have that

$$
\begin{aligned}
\left\|\vec{f}_{k}\right\|_{L^{p}(W)}^{p} & =\left\|W^{\frac{1}{p}} \vec{f}_{k}\right\|_{L^{p}}^{p} \\
& =\left\|\left(W^{\frac{1}{p}} M_{W, p}^{-1}\right) M_{W, p} \vec{f}_{k}\right\|_{L^{p}}^{p} \\
& \lesssim\|W\|_{\mathrm{A}_{p}}^{1+\lceil p\rceil}\left\|M_{W, p} \vec{f}_{k}\right\|_{L^{p}}^{p} \\
& \lesssim\|W\|_{\mathrm{A}_{p}}^{1+\lceil p\rceil} \int_{\mathbb{R}^{d}}\left(\sum_{I \in \mathscr{D}} \sum_{\varepsilon \in \operatorname{Sig}_{d}} \frac{\left|V_{I} \vec{f}_{I}^{\varepsilon}\right|^{2}}{|I|} \chi_{I}(x)\right)^{\frac{p}{2}} d x
\end{aligned}
$$

by standard dyadic Littlewood-Paley theory. However, since $\vec{f} \in C_{c}^{\infty}\left(\mathbb{R}^{d}\right)$ we have that $\overrightarrow{f_{k}} \rightarrow \vec{f}$ pointwise, so finally by Fatou's lemma

$$
\begin{aligned}
\|\vec{f}\|_{L^{p}(W)}^{p} & \leq \liminf _{k \rightarrow \infty}\left\|\vec{f}_{k}\right\|_{L^{p}(W)}^{p} \\
& \lesssim\|W\|_{\mathrm{A}_{p}}^{1+\lceil p\rceil} \int_{\mathbb{R}^{d}}\left(\sum_{I \in \mathscr{D}} \sum_{\varepsilon \in \operatorname{Sig}_{d}} \frac{\left|V_{I} \vec{f}_{I}^{\varepsilon}\right|^{2}}{|I|} \chi_{I}(x)\right)^{\frac{p}{2}} d x
\end{aligned}
$$

Now we prove the second inequality in Theorem 1.1 for $C_{c}^{\infty}\left(\mathbb{R}^{d}\right)$ functions. If $\vec{f} \in C_{c}^{\infty}\left(\mathbb{R}^{d}\right)$ then let $\vec{\Psi}_{k}$ be defined by

$$
\vec{\Psi}_{k}=\sum_{I \in F_{k}} \sum_{\varepsilon \in \operatorname{Sig}_{d}} V_{I}^{-1} \vec{f}_{I}^{\varepsilon} h_{I}^{\varepsilon} .
$$

Also for $\vec{g} \in \mathscr{F}$, define $\vec{g}_{k}$ as before and let $F_{\vec{g}}$ be the class of $I \in \mathscr{D}$ such that the Haar coefficient of $\vec{g}$ with respect to $I$ is not zero. Then clearly

$$
\begin{aligned}
& \left|\left\langle\vec{\Psi}_{k}, \vec{g}\right\rangle_{L^{2}}\right|=\left|\sum_{I \in F_{k} \cap F_{\vec{g}}} \sum_{\varepsilon \in \operatorname{Sig}_{d}}\left\langle V_{I}^{-1} \vec{f}_{I}^{\varepsilon}, \vec{g}_{I}^{\varepsilon}\right\rangle_{\mathbb{C}^{n}}\right| \\
& =\left|\sum_{I \in \mathscr{D}} \sum_{\varepsilon \in \operatorname{Sig}_{d}}\left\langle\vec{f}_{I}^{\varepsilon}, V_{I}^{-1}\left(\vec{g}_{k}\right)_{I}^{\varepsilon}\right\rangle_{\mathbb{C}^{n}}\right| \\
& \leq \int_{\mathbb{R}^{d}}\left|\left\langle\vec{f}, M_{W, p}^{-1} \vec{g}_{k}\right\rangle_{\mathbb{C}^{n}}\right| d x \\
& =\int_{\mathbb{R}^{d}}\left|\left\langle W^{-\frac{1}{p}} \vec{f}, W^{\frac{1}{p}} M_{W, p}^{-1} \vec{g}_{k}\right\rangle_{\mathbb{C}^{n}}\right| d x \\
& \lesssim\|W\|_{\mathrm{A}_{p}}^{\frac{1}{p}+\frac{\lceil p\rceil}{p}}\left\|W^{-\frac{1}{p}} \vec{f}\right\|_{L^{p^{\prime}}}\|\vec{g}\|_{L^{p}}
\end{aligned}
$$

By a trivial approximation argument we therefore have

$$
\sup _{k}\left\|\vec{\Psi}_{k}\right\|_{L^{p^{\prime}}} \lesssim\|W\|_{\mathrm{A}_{p}}^{\frac{1}{p}+\frac{\Gamma p\rceil}{p}}\left\|W^{-\frac{1}{p}} \vec{f}\right\|_{L^{p^{\prime}}}
$$

which by dyadic Littlewood-Paley theory and the monotone convergence theorem says 


$$
\begin{aligned}
& \left(\int_{\mathbb{R}^{d}}\left(\sum_{I \in \mathscr{D}} \sum_{\varepsilon \in \operatorname{Sig}_{d}} \frac{\left|V_{I}^{-1} \vec{f}_{I}^{\varepsilon}\right|^{2}}{|I|} \chi_{I}(x)\right)^{\frac{p^{\prime}}{2}} d x\right)^{\frac{1}{p^{\prime}}} \\
& =\lim _{k \rightarrow \infty}\left(\int_{\mathbb{R}^{d}}\left(\sum_{I \in F_{k}} \sum_{\varepsilon \in \operatorname{Sig}_{d}} \frac{\left|V_{I}^{-1} \vec{f}_{I}^{\varepsilon}\right|^{2}}{|I|} \chi_{I}(x)\right)^{\frac{p^{\prime}}{2}}\right)^{\frac{1}{p^{\prime}}} \\
& \lesssim \sup _{k}\left\|\vec{\Psi}_{k}\right\|_{L^{p^{\prime}}} \\
& \lesssim\|W\|_{\mathrm{A}_{p}}^{\frac{1}{p}+\frac{[p]}{p}}\left\|W^{-\frac{1}{p}} \vec{f}\right\|_{L^{p^{\prime}}}
\end{aligned}
$$

which means that

$$
\begin{aligned}
& \left(\int_{\mathbb{R}^{d}}\left(\sum_{I \in \mathscr{D}} \sum_{\varepsilon \in \mathrm{Sig}_{d}} \frac{\left|V_{I}^{\prime} \vec{f}_{I}^{\varepsilon}\right|^{2}}{|I|} \chi_{I}(x)\right)^{\frac{p^{\prime}}{2}} d x\right)^{\frac{1}{p^{\prime}}} \\
& \leq\|W\|_{\mathrm{A}_{p}}^{\frac{1}{p}}\left(\int_{\mathbb{R}^{d}}\left(\sum_{I \in \mathscr{D}} \sum_{\varepsilon \in \operatorname{Sig}_{d}} \frac{\left|V_{I}^{-1} \vec{f}_{I}^{\varepsilon}\right|^{2}}{|I|} \chi_{I}(x)\right)^{\frac{p^{\prime}}{2}} d x\right)^{\frac{1}{p^{\prime}}} \\
& \lesssim\|W\|_{\mathrm{A}_{p}}^{\frac{2}{p}+\frac{\lceil p\rceil}{p}}\left\|W^{-\frac{1}{p}} \vec{f}\right\|_{L^{p^{\prime}}}
\end{aligned}
$$

by the matrix $\mathrm{A}_{p}$ condition. However, $W$ is a matrix $\mathrm{A}_{p}$ weight if and only if $W^{1-p^{\prime}}$ is a matrix $\mathrm{A}_{p^{\prime}}$ weight with $\left\|W^{1-p^{\prime}}\right\|_{\mathrm{A}_{p^{\prime}}}=\|W\|_{\mathrm{A}_{p}}^{\frac{1}{p-1}}$. Furthermore, one can easily check that $V_{I}^{\prime}\left(W^{1-p^{\prime}}, p^{\prime}\right)=V_{I}(W, p)$ which then means that

$$
\begin{aligned}
& \left(\int_{\mathbb{R}^{d}}\left(\sum_{I \in \mathscr{D}} \sum_{\varepsilon \in \operatorname{Sig}_{d}} \frac{\left|V_{I} \vec{f}_{I}^{\varepsilon}\right|^{2}}{|I|} \chi_{I}(x)\right)^{\frac{p}{2}} d x\right)^{\frac{1}{p}} \\
& \lesssim\left\|W^{1-p^{\prime}}\right\|_{\mathrm{A}_{p^{\prime}}}^{\frac{2}{p^{\prime}}+\frac{\left\lceil p^{\prime}\right\rceil}{p^{\prime}}}\|\vec{f}\|_{L^{p}(W)} \\
& =\|W\|_{\mathrm{A}_{p}}^{\frac{2}{(p-1) p^{\prime}}+\frac{\left\lceil p^{\prime}\right\rceil}{(p-1) p^{\prime}}}\|\vec{f}\|_{L^{p}(W)} \\
& =\|W\|_{\mathrm{A}_{p}}^{\frac{2}{p}+\frac{\left\lceil p^{\prime}\right\rceil}{p}}\|\vec{f}\|_{L^{p}(W)}
\end{aligned}
$$

which completes the proof for $C_{c}^{\infty}\left(\mathbb{R}^{d}\right)$ functions.

Finally we will complete the proof of Theorem 1.1 for $\vec{f} \in L^{p}(W)$. Pick a sequence $\left\{\vec{f}_{k}\right\}_{k=1}^{\infty} \subseteq C_{c}^{\infty}\left(\mathbb{R}^{d}\right)$ where $\vec{f}_{k} \rightarrow f$ in $L^{p}(W)$. Note that since $W^{1-p^{\prime}}$ is locally integrable we clearly have that $\left(\vec{f}_{k}\right)_{I}^{\varepsilon} \rightarrow \vec{f}_{I}^{\varepsilon}$ for any $I \in \mathscr{D}$ and $\varepsilon \in \operatorname{Sig}_{d}$. Then by Theorem 1.1 for $C_{c}^{\infty}\left(\mathbb{R}^{d}\right)$ functions, Fatou's lemma, and the two facts 
stated above, we have

$$
\begin{aligned}
\|W\|_{\mathrm{A}_{p}}^{2+\left\lceil p^{\prime}\right\rceil}\|\vec{f}\|_{L^{p}(W)}^{p} & =\|W\|_{\mathrm{A}_{p}}^{2+\left\lceil p^{\prime}\right\rceil} \liminf _{k \rightarrow \infty}\left\|\vec{f}_{k}\right\|_{L^{p}(W)}^{p} \\
& \gtrsim \liminf _{k \rightarrow \infty} \int_{\mathbb{R}^{d}}\left(\sum_{I \in \mathscr{D}} \sum_{\varepsilon \in \operatorname{Sig}_{d}} \frac{\left|V_{I}\left(\vec{f}_{k}\right)_{I}^{\varepsilon}\right|^{2}}{|I|} \chi_{I}(x)\right)^{\frac{p}{2}} d x \\
& \geq \int_{\mathbb{R}^{d}}\left(\sum_{I \in \mathscr{D}} \sum_{\varepsilon \in \operatorname{Sig}_{d}} \frac{\left|V_{I} \vec{f}_{I}^{\vec{\varepsilon}}\right|^{2}}{|I|} \chi_{I}(x)\right)^{\frac{p}{2}} d x
\end{aligned}
$$

which proves half of Theorem 1.1 for $\vec{f} \in L^{p}(W)$, and additionally proves that

$$
\lim _{k \rightarrow \infty} \int_{\mathbb{R}^{d}}\left(\sum_{I \in \mathscr{D}} \sum_{\varepsilon \in \operatorname{Sig}_{d}} \frac{\left|V_{I}\left(\overrightarrow{f_{k}}-\vec{f}\right)_{I}^{\varepsilon}\right|^{2}}{|I|} \chi_{I}(x)\right)^{\frac{p}{2}} d x=0 .
$$

The other half now follows immediately since

$$
\begin{aligned}
\|\vec{f}\|_{L^{p}(W)}^{p} & =\lim _{k \rightarrow \infty}\left\|\vec{f}_{k}\right\|_{L^{p}(W)}^{p} \\
& \lesssim\|W\|_{\mathrm{A}_{p}}^{1+\lceil p\rceil} \lim _{k \rightarrow \infty} \int_{\mathbb{R}^{d}}\left(\sum_{I \in \mathscr{D}} \sum_{\varepsilon \in \operatorname{Sig}_{d}} \frac{\mid V_{I}\left(\vec{f}_{k}\right)_{I}^{\varepsilon}}{|I|} \chi_{I}(x)\right)^{\frac{p}{2}} d x \\
& =\|W\|_{\mathrm{A}_{p}}^{1+\lceil p\rceil} \int_{\mathbb{R}^{d}}\left(\sum_{I \in \mathscr{D}} \sum_{\varepsilon \in \operatorname{Sig}_{d}} \frac{\left|V_{I} \vec{f}_{I}^{\varepsilon}\right|^{2}}{|I|} \chi_{I}(x)\right)^{\frac{p}{2}} d x
\end{aligned}
$$

Let us finish this paper with two comments. As was earlier commented, Lemma 2.4 holds for matrix $\mathrm{A}_{p, \infty}$ weights. Because of this, the attentive reader will notice that we have actually proved the following, which was also proved in [10,15] when $d=1$.

Theorem 3.1. If $W$ is a matrix $A_{p, \infty}$ weight and $1<p<\infty$ then

$$
\left(\int_{\mathbb{R}^{d}}\left(\sum_{I \in \mathscr{D}} \sum_{\varepsilon \in S i g_{d}} \frac{\left|V_{I}^{-1} \vec{f}_{I}^{\varepsilon}\right|^{2}}{|I|} \chi_{I}(x)\right)^{\frac{p^{\prime}}{2}} d x\right)^{\frac{1}{p^{\prime}}} \lesssim\|\vec{f}\|_{L^{p^{\prime}}\left(W^{1-p^{\prime}}\right)}
$$

Finally, note that when $p=2$ and $W$ is a matrix $\mathrm{A}_{2}$ weight, our results considerably simplify to the inequalities

$$
\|\vec{f}\|_{L^{2}(W)} \lesssim\|W\|_{\mathrm{A}_{2}}^{\frac{3}{2}}\left(\sum_{I \in \mathscr{D}} \sum_{\varepsilon \in \operatorname{Sig}_{d}}\left|\left(m_{I} W\right)^{\frac{1}{2}} \vec{f}_{I}^{\varepsilon}\right|^{2}\right)^{\frac{1}{2}}
$$

and

$$
\left(\sum_{I \in \mathscr{D}} \sum_{\varepsilon \in \operatorname{Sig}_{d}}\left|\left(m_{I} W\right)^{\frac{1}{2}} \vec{f}_{I}^{\varepsilon}\right|^{2}\right)^{\frac{1}{2}} \lesssim\|W\|_{\mathrm{A}_{2}}^{2}\|\vec{f}\|_{L^{2}(W)} .
$$


Note that these "square function bounds" were obtained first in [11] (where the $\mathrm{A}_{2}$ characteristic dependence was not tracked) also using stopping techniques that are similar to the ones in 9]. Moreover, these square function bounds were proved in [1,

3] using vastly different techniques but with the better bounds of $\|W\|_{\mathrm{A}_{2}}^{\frac{1}{2}}\left(\log \|W\|_{\mathrm{A}_{2}}\right)^{\frac{1}{2}}$ and $\|W\|_{\mathrm{A}_{2}}\left(\log \|W\|_{\mathrm{A}_{2}}\right)^{\frac{1}{2}}$, respectively. Finally, it is well known and was proved in 66, 12] that in the scalar $\mathrm{A}_{2}$ setting the bounds of $\|W\|_{\mathrm{A}_{2}}^{\frac{1}{2}}$ and $\|W\|_{\mathrm{A}_{2}}$ are sharp. It appears to be a very interesting but challenging problem as to whether these bounds hold in the matrix weighted setting.

\section{REFERENCES}

[1] K. Bickel, S. Petermichl, and B Wick, Bounds for the Hilbert Transform with Matrix $A_{2}$ Weights, Preprint available at http://arxiv.org/abs/1402.3886

[2] D. Cruz-Uribe SFO, K. Moen, and S. Rodney, Matrix $A_{p}$ weights, degenerate Sobolev spaces, and mappings of finite distortion, Preprint available at http://arxiv.org/pdf/1505.00699.pdf.

[3] A. Culiuc and B. Wick, Preprint.

[4] M. Frazier, B. Jawerth, and G. Weiss, Littlewood-Paley theory and the study of function spaces, CBMS Regional Conference Series in Mathematics, 1991. MR1107300

[5] M. Goldberg, Matrix $A_{p}$ weights via maximal functions, Pacific J. Math. 211 (2003), 201 220. MR2015733

[6] S. Hukovic, S. Treil, and A. Volberg, The Bellman functions and sharp weighted inequalities for square functions, Oper. Theory Adv. Appl., 113 (2003), 97 - 113. MR1771755

[7] J. Isralowitz, A Matrix weighted T1 theorem for matrix kernelled CZOs, Preprint.

[8] J. Isralowitz, H. K. Kwon, and S. Pott, Matrix weighted norm inequalities for commutators and paraproducts with matrix symbols, Preprint available at http://arxiv.org/abs/1507.04032.

[9] N. H. Katz and M. C. Pereyra, Haar multipliers, paraproducts, and weighted inequalities, Appl. Numer. Harmon. Anal. Analysis of divergence (1997), 145 - 170. MR1731264

[10] F. Nazarov and S. Treil, The hunt for a Bellman function: applications to estimates for singular integral operators and to other classical problems of harmonic analysis, Algebra $\mathrm{i}$ Analiz 8 (1996), 32 - 162. MR1428988

[11] S. Pott, A sufficient condition for the boundedness of operator-weighted martingale transforms and Hilbert transform, Studia Math. 182 (2007), 99 - 111. MR2338479

[12] S. Petermichl and S. Pott, An estimate for weighted Hilbert transform via square functions, Trans. Amer. Math. Soc. 354 (2002), 1699 - 1703. MR1873024

[13] S. Roudenko, Matrix-weighted Besov spaces, Trans. Amer. Math. Soc. 355 (2003), 273 - 314. MR1928089

[14] S. Treil and A. Volberg, Wavelets and the angle between past and future, J. Funct. Anal. 143 (1997), 269 308. MR1428818

[15] A. Volberg, Matrix $A_{p}$ weights via S-functions, J. Amer. Math. Soc. 10 (1997), 445 - 466. MR1423034

(Joshua Isralowitz) Department of Mathematics and Statistics, SUNy Albany, 1400 Washington Ave., Albany, NY, 12222

E-mail address, Joshua Isralowitz: jisralowitz@albany.edu 